\title{
Cationic Core/Shell Polysiloxane Acrylate Emulsion: Synthesis, Film Morphology, and Performance on Cotton Pigment Coloration.
}

\section{Xianghui Dai}

Donghua University - Songjiang Campus: Donghua University

xia Yu

Donghua University - Songjiang Campus: Donghua University

\section{Xiaomin Xu}

Donghua University - Songjiang Campus: Donghua University

\section{Xiao Sun}

Donghua University - Songjiang Campus: Donghua University

\section{Jianjun Pan}

Huzhou customs

\section{Xiaoting Zhang}

Huzhou customs

Jie Min ( $\nabla$ minjie@dhu.edu.cn )

Donghua University - Songjiang Campus: Donghua University https://orcid.org/0000-0001-7073-1229

\section{Research Article}

Keywords: cation, core / shells / shelled structure, silicon modification, poly acrylate, pigment dyeing.

Posted Date: September 23rd, 2021

DOl: https://doi.org/10.21203/rs.3.rs-819618/v1

License: (9) This work is licensed under a Creative Commons Attribution 4.0 International License. Read Full License

Version of Record: A version of this preprint was published at Cellulose on January 4th, 2022. See the published version at https://doi.org/10.1007/s10570-021-04396-3. 


\title{
Cationic Core/Shell Polysiloxane Acrylate Emulsion: Synthesis, Film
}

\section{Morphology, and Performance on Cotton Pigment Coloration.}

\author{
Xianghui Dai ${ }^{1, a}$, Xia Yu ${ }^{\mathrm{a}}$, Xiaomin $\mathrm{Xu}^{\mathrm{a}}$, Xiao Sun ${ }^{\mathrm{a}}$, Jianjun Pan $^{\mathrm{c}}$, Xiaoting Zhang ${ }^{\mathrm{c}}$, \\ Jie $\operatorname{Min}^{\mathrm{a}, \mathrm{b}}$ * \\ a. College of Chemistry, Chemical Engineering and Biotechnology, Donghua University, \\ Shanghai 201620, China \\ b. Key Laboratory of Textile Science \& Technology, Ministry of Education, Shanghai \\ 201620, China \\ c. Huzhou Customs, Zhejiang Huzhou, 313000, China
}

\begin{abstract}
In this paper, acrylate-terminated polysiloxane was synthesised and used as raw materials for the synthesis of cationic core/shell polysiloxane acrylate emulsion, which was applied as binders in the pigment dyeing of cotton fabrics.

The experimental results showed that: the cationic core/shell poly siloxane acrylate emulsion in this article is in nano size of $100 \mathrm{~nm}$. During the film formation of the emulsion, there are core-core and shell-shell fusion phenomena between the latex particles, and same poly siloxane chain aggregates on the surface of the film. When the emulsion is used in the pigment dyeing of cotton fabrics, the dyed cotton fabrics feel soft, and the soaping fastness and dry/wet rubbing fastness meet the national quality requirements, as soaping fastness can reach grade $4 \sim 5$, dry rubbing fastness can reach grade 4 5, and wet rubbing fastness can reach grade 4 .
\end{abstract}

Keywords: cation; core / shells / shelled structure; silicon modification; poly acrylate; pigment dyeing.

\section{Introduction}

The printing and dyeing industry is a resource-dependent and environmentally sensitive industry. With raising public's attention to energy and environmental issues, its development is constrained by more laws and regulations. How to achieve clean 
production, energy-saving, and emission reduction is the key to improve the competitiveness of enterprises, and also the starting point in the survival and development of the printing and dyeing industry.

Pigments have been widely used in the textile printing for a long time, and its patents and research reports were not published until the 1960s (Womble 1952). Now pigment dyeing has been accepted by producers and consumers in most countries but in China it began to be studied, applied and gradually started the mass production in 1980s (Song 2013a; Song 2013b; Song 2013c). With the emergence of new type binders, auxiliaries, and pigment dyeing technologies, this dyeing method has gained more attention in China's printing and dyeing industry.

Unlike dyes, the pigment is an insoluble colored substance and difficult to enter the fiber as it has no affinity to the fiber and cannot be used to dye the fiber directly. Pigment dyeing is a dispersion system of pigments and binders, which is applied evenly to the fabric. The binder in the dispersion system bonds the pigments firmly to the fabric surface (Sun and Li. 2007). Compared with traditional dye dyeing, pigment dyeing has many advantages: pigment is not selective to fiber, suitable for dyeing of various fiber (including glass fiber, etc..) and multi-component fiber dyeing, complete chromatogram, convenient color matching, and short processing flow, energy saving, water saving, etc.. The main problems of pigment dyeing are: rough handle of dyed fabrics, difficulty to dye dark colors, low rubbing fastness, especially the wet rubbing fastness of dark varieties etc (Jie 2005).

In order to improve the cotton fabrics pigment dyeing quality, the main research methods are the cationic modification of cotton fiber (Fang K 2010; J M Xia 1996; Lewis and Mcllroy 2010; Yaman N 2012), ultra-fine processing of pigment color paste (C X Wang 2009; Fu et al. 2013; Sun and Li. 2007; Xu 2009), and structural design of binder (F Y Shen 2009; Ishwarlal 2000; J L Ji 2009; Martini et al. 1991; Martini et al. 1993). The pre-modification of cotton fiber is to treat the fabric with the cationic modifier to get the fabric surface positive charged (Wu and Zhang 2013), which is followed by pigment dyeing. The dyeing depth and fastness of the dyed fabric can be improved by increasing the electrostatic attraction between the fiber with the binder 
and pigment. Pigment dyeing binders are used as film-forming substances in dyeing prescriptions and the binder's properties play an important role in the fastness and hand feel of dyed fabrics. Therefore, the design and synthesis of new binders is of the most concern to researchers (H Li 2013; Song 2015; Sui et al. 2013; Wu 2016). Research shows that cationic polyacrylate binders used for pigment dyeing are better than conventional anionic binders in color yield and rubbing fastness (J L Ji 2009).

This article uses self-made acrylate-terminated polysiloxane as raw materials to synthesize cationic core/shell polysiloxane acrylate emulsion and also uses it as a binder in the pigment dyeing to cotton fabric. The particle structure and film properties of synthetic emulsions, as well as their effects on the color fastness and hand feeling of cotton fabric are investigated.

\section{Material and methods}

\subsection{Reagents and instruments}

Reagents:

n-butyl acrylate, methyl methacrylate, acrylamide, cetyltrimethylammonium bromide, span 80 (directly used), and azodiisobutymidine hydrochloride were provided by Sinopharm Chemical Reagent Co., Ltd. (chemical purity); blocked polyisocyanate ester, industrial grade, Shanghai Beike Chemical Co., Ltd.; epoxy-terminated polysiloxane $(\mathrm{M}=1000)$, Anhui Keguang New Materials Co., Ltd.; pigment blue dyeing pulp (B31, non-ionic, $\mathrm{d}=130 \mathrm{~nm}$, grinding and dispersing in our laboratory); pure cotton plain weave bleached fabric $\left(124 \mathrm{~g} / \mathrm{m}^{2}, 82 / 2 * 80 / 2\right.$, Zhong Heng Da Yao Textile Technology Co., Ltd.).

Instruments:

constant temperature heated magnetic stirrer DF-101s (Gongyi Yuhua Instrument Co., Ltd.), nanoparticle size analyzer Zetasizer nano ZS (Malvern Instrument Co., Ltd.), transmission electron microscope JEOL TEM-2100 (Nippon Electronics Co., Ltd.), scanning electron microscope TM-1000 (Hita-Chi Co., Ltd.), x-ray atomic energy 
spectrometer (XPS)Escalab 250xi (Thermo Fisher Technology Co., Ltd.), contact angle meter DSA30（Klux, Germany), colorimeter Datacolor650 (Delta color system (Asia Pacific) Co., Ltd.), surface contact angle analyzer LSA (KRÜSS), fabric heat setting machine UPEI (Taiwan (Xiamen) Rabbi Co., Ltd.), friction fastness tester Y571b (Wenzhou Textile Instrument Factory), stiffness tester SDL-ATLAS (Ningbo Textile Instrument Factory).

\subsection{Preparation of acrylate-terminated polysiloxane}

2.2.1 Determination of epoxy value of epoxy-terminated silicones oil (Tong 2012).

Weigh $1.0 \mathrm{~g}$ (accurate to $0.0001 \mathrm{~g}$ ) ended epoxy silicone oil in a $250 \mathrm{~mL}$ iodine measuring flasks, add $25 \mathrm{~mL}$ of hydrochloric acid-acetone solution to a pipette, cover and shake to make the silicone oil completely dissolved. Set it in a cool place for about $15 \mathrm{~min}$; then add 3-5 drops of phenolphthalein indicator and titrate with a $0.2 \mathrm{~mol} / \mathrm{L}$ sodium hydroxide standard solution to pink. The color lasts for $15 \mathrm{~s}$, which is the end of the titration. Calculate the epoxy value of epoxy-terminated silicone oil according to the following formula (1). Take two parallel samples, repeat the measurement 3 times, and take the average of the 3 measurements. At the same time, make a blank experiment, and also make two parallels samples, then take the average of 3 measurements. Calculate according to the following formula:

$$
E v=\frac{C(V 0-V)}{m \times 10}
$$

Where, $\mathrm{EV}_{\mathrm{V}}$ is the epoxy value of the epoxy-terminated silicone oil mol/100 $\mathrm{g}, \mathrm{C}$ is the concentration of the sodium hydroxide standard solution $0.2 \mathrm{~mol} / \mathrm{L}, \mathrm{V} 0$ and $\mathrm{V}$ are the blank sample and the sample consumption sodium hydroxide standard solution volume respectively $(\mathrm{mL}), \mathrm{m}$ is the mass of the sample $(\mathrm{g})$, respectively. Through experiment and calculation, the epoxy value of the epoxy-terminated silicone oil raw material is $0.1780 \mathrm{~mol} / 100 \mathrm{~g}$.

\subsubsection{Preparation of acrylate-terminated silicone oil}

Weigh a certain amount of terminal epoxy polysiloxane and an appropriate amount of triethylamine catalyst into a four-neck flask, heat it to $95^{\circ} \mathrm{C}$ in oil bath, then a mixture 
of acrylic acid equimolar was added into an epoxy group and polymerization inhibitor p-hydroxyanisole in dropwise for $2 \mathrm{~h}$. After that, the temperature is raised to $110^{\circ} \mathrm{C}$, and keep it heated for $3 \mathrm{~h}$. When the acid value of the reactant is less than $3 \mathrm{mg} \mathrm{KOH} / \mathrm{g}$, the end of the reaction is reached. After the temperature is below room temperature, wash with $2 \%$ sodium carbonate solution twice, then wash with deionized water three times to obtain acrylate-terminated polysiloxane.

2.3 Synthesis of cationic core/shell polysiloxane acrylate emulsion

The polymerization process of cationic core/shell polysiloxane acrylate emulsion is shown in Fig. 1

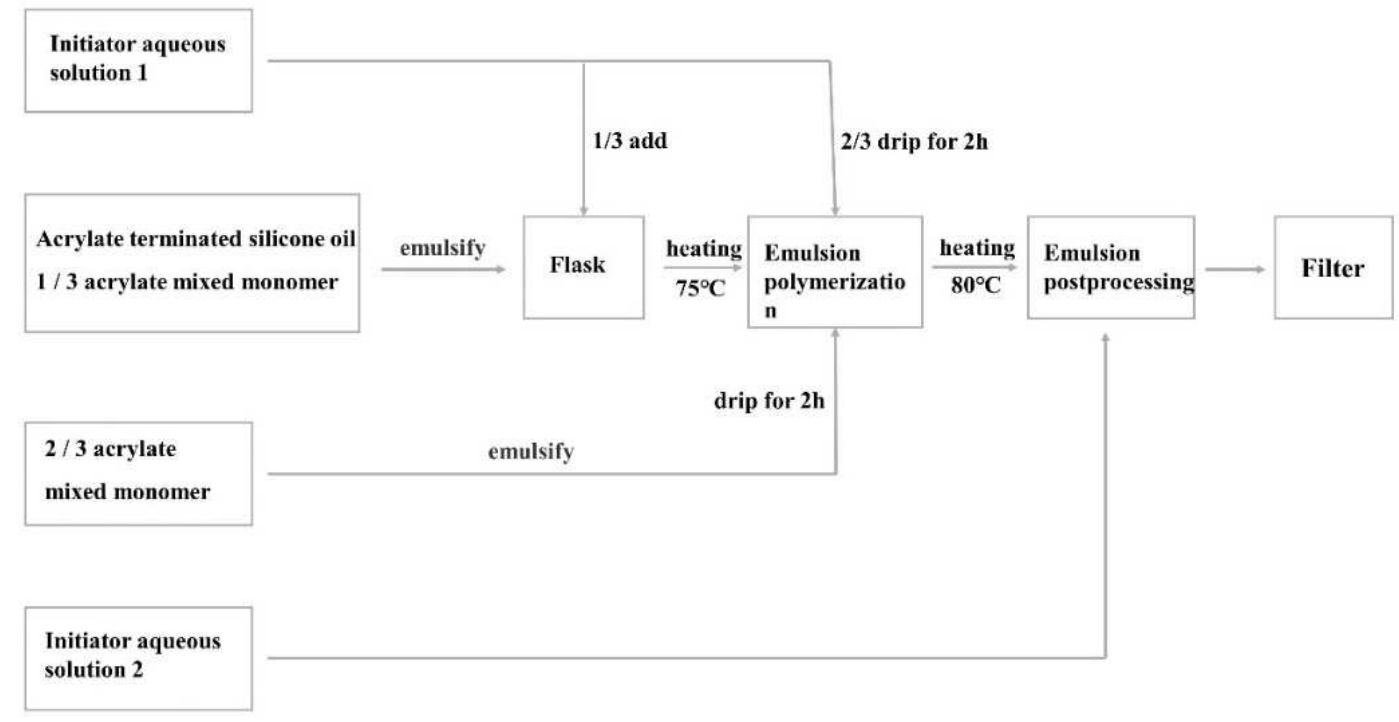

Fig.1 Emulsion polymerization process

Initiator aqueous solution:

Initiator aqueous solution 1 (azobisisobutamidine hydrochloride) is $0.7 \%$ by weight of the acrylate monomer, dissolved in $20 \mathrm{~mL}$ of deionized water; Initiator aqueous solution 2 (azobisisobutamidine hydrochloride) is $0.1 \%$ of the weight of the acrylate monomer, dissolved in $10 \mathrm{~mL}$ of deionized water, and the initiator aqueous solution placed in a constant pressure dropper for later use.

Emulsification: Take acrylate-terminated polysiloxane and 1/3 of the acrylate mixed monomer, add deionized water and emulsifier (hexadecyltrimethylammonium 
bromide: Span 80=2:1 (weight ratio)), emulsify with a homogenizer at a rate of 10000 r/min for $15 \mathrm{~min}$, and transferred to a four-neck flask as bottom emulsion polymerization; Use the same emulsification method to emulsify the remaining $2 / 3$ acrylate mixed monomer, and transferred to the corresponding constant pressure dropper.

Polymerization of cationic core/shell polysiloxane acrylate emulsion:

The four-neck flask is equipped with a polytetrafluoroethylene stirring blade, two constant pressure dropping funnels, and a thermometer. After adding the polymerization reaction bottom emulsion, add 1/3 of the polymerization initiator 1 aqueous solution, and the temperature of the reaction system was heated to $75^{\circ} \mathrm{C}$ with $250 \mathrm{r} / \mathrm{min}$ stirring speed. As the reaction is kept warm until the emulsion shows blue light, the remaining $2 / 3$ of the initiator aqueous solution and $2 / 3$ of the acrylate mixed monomer emulsion are added dropwise to the emulsion at a uniform rate. The dripping time is $2 \mathrm{~h}$ and keep the reaction for another $2 \mathrm{~h}$. Adjust the $\mathrm{pH}$ value of the emulsion to about 5-6 with acetic acid and add initiator 2, keep the reaction for $2 \mathrm{~h}$ in $80{ }^{\circ} \mathrm{C}$. After the emulsion is cooled to room temperature, filtered with a 200-mesh screen, a cationic core/shell polysiloxane acrylate emulsion is obtained. The emulsion polymerization reaction formula is shown in Fig 2.

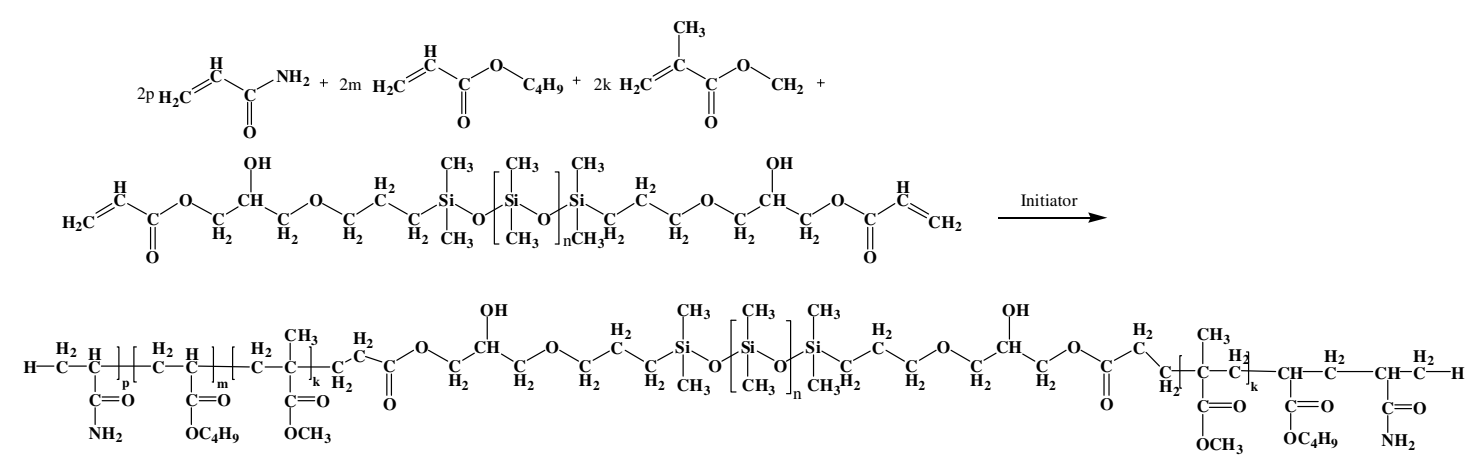

Fig.2 Emulsion polymerization reaction equation

\subsection{Pigment dyeing process and formulation}

Pigment pad dyeing process:

Cotton fabric $\rightarrow$ Two dipping/padding ( $85 \%$ with liquid rate) Pigment dyeing 
solution $\rightarrow$ pre-drying $\left(80^{\circ} \mathrm{C}, 3 \mathrm{~min}\right) \rightarrow$ baking $\left(140^{\circ} \mathrm{C}, 3 \mathrm{~min}\right)$

Pigment dyeing formula: $(\mathrm{g} / \mathrm{L})$

Pigment blue (B31 non-ionic, self-made). 2

Cationic core/shell polysiloxane acrylate emulsion $\quad \mathrm{X}$

Crosslinking agent (Waterborne blocked isocyanate). 2

$\begin{array}{ll}\text { Total } & 1000\end{array}$

\subsection{Examination}

\subsubsection{Emulsion performance}

Dilution stability: Dilute $2 \mathrm{~mL}$ emulsion sample into 10 times, and leave it for 48 $\mathrm{h}$ in the constant temperature and humidity room. Observe whether the solution precipitates or separate.

Particle size: Dilute $1 \mathrm{~mL}$ emulsion sample into 500 times, and test particle size and distribution with a Zetasizer Nano ZS nanoparticle size analyzer.

Characterization of the morphology of latex particles: Dilute $2 \mathrm{~mL}$ emulsion sample into 250 times, dye it with phosphotungstic acid, drop it on a copper net, leave it for $3 \mathrm{~min}$, and then absorb the excess liquid with filter paper. After natural drying, the samples were tested with a JEOL TEM- 2100 transmission electron microscope.

Solid content: A sample of M1 g emulsion was dried to a constant weight in an oven at $105{ }^{\circ} \mathrm{C}$. The mass of the sample after drying is M2 g. Calculate according to the following formula:

$$
X=\frac{M_{2}}{M_{1}} \times 100 \%
$$

Where: X -- solid content of emulsion sample (\%), M1 -- sample mass before drying (g), M2 -- sample mass after drying (g), respectively.

Monomer conversion rate: The monomer conversion rate is calculated by dividing the actual solid content of the solution by the theoretical solid content. The calculation formula is as follows:

$$
C=\frac{X}{\frac{M_{3}}{M_{3}+M_{4}}} \times 100 \%
$$

Where: $\mathrm{C}$-- total conversion rate of monomer (\%), $\mathrm{X}$-- solid content of emulsion 
measured (\%), M3 - the total mass of monomer in polymerization system (g), M4 - the mass of other non-monomer media $(\mathrm{g})$, respectively.

\subsubsection{Performance analysis of emulsion film}

Contact angle: Place 5mL emulsion sample on a polytetrafluoroethylene template and dry it to form a film at room temperature, then dry it at $105^{\circ} \mathrm{C}$ for $20 \mathrm{~min}$. Use the contact angle tester (LSA KRÜSS) to test the film water contact angle.

Hygroscopicity of polymer emulsion film: Dry $5 \mathrm{~mL}$ emulsion sample at room temperature to form a film, then dry it at $105^{\circ} \mathrm{C}$ to a constant weight and weigh it as M5 $\mathrm{g}$, then place the film in deionized water for $48 \mathrm{~h}$, excess water is absorbed from the surface of the film with absorbent paper and weigh it as M6 g. The Hygroscopicity of the film is calculated according to the following formula:

$$
W=\frac{(M 6-M 5)}{M 5} \times 100 \%
$$

Where: W — hygroscopicity of the film (\%), M5 — the dry weight of the sample (g), M6 - the weight of the sample after immersion in water $(\mathrm{g})$.

Mechanical properties of the film: After drying the emulsion sample into a film, put it into the oven at $105^{\circ} \mathrm{C}$ for $20 \mathrm{~min}$. Cut the film into rectangular strips with $5 \mathrm{~cm}$ in length and $1 \mathrm{~cm}$ in width. Place the rectangular strip of the film in a constant temperature $\left(20^{\circ} \mathrm{C}\right)$ and humidity $(\mathrm{RH}=65 \%)$ room for $24 \mathrm{~h}$. Select 3 points on the film and measure the thickness and width. The universal material testing machine is used to measure the tensile strength and elongation at the break of the film. Set the tensile rate to $150 \mathrm{~mm} / \mathrm{min}$. The tensile strength of the film is calculated according to the following formula:

$$
P=\frac{F}{S}
$$

Where, $\mathrm{P}$-film tensile breaking strength $(\mathrm{Pa}), \mathrm{F}$-load value when the membrane is $\operatorname{broken}(\mathrm{N}), \mathrm{S}$ - the cross-sectional area of the membrane $\left(\mathrm{m}^{2}\right)$.

The film breaking elongation is calculated as follows:

$$
X=\frac{L-L 0}{L 0} \times 100 \%
$$

Where, $\mathrm{X}$ - fracture elongation(\%), L — the stretched length when the film is broken 
(mm), $\mathrm{L}_{0}-$ the original length of the membrane $(\mathrm{mm})$.

\subsubsection{Pigment dyeing application performance}

Color fastness to rubbing(dry/wet): The color fastness to rubbing of the dyed fabric is measured with the Y571L rubbing and scrubbing color fastness tester. The samples are rated according to ISO 105-X12-2001 "Color fastness to rubbing".

Soaping fastness: Carry out the soaping test according to ISO 105-C10:2006 standard. According to ISO105-A02:1993 gray sample card for color change and ISO 105-A03:1993 gray sample card for staining, under D65 standard light source, the staining fastness of the sample and the lining fabric are rated.

K/S value: Using a Datacolor color matcher with a D65 light source and a $10^{\circ}$ viewing angle, measure 5 points on the fabric surface, and calculate the average value.

Fabric softness: The stiffness and softness of the fabric are a pair of opposite hand feeling effects. This article will use the stiffness data to characterize the softness of the fabric, that is, the lower the stiffness of the fabric, the better the softness of the fabric.

Test the stiffness of the fabric in accordance with ASTM/D1388-96 "Standard Test Method for Fabric Stiffness". The fabric is cut into $20 \mathrm{~cm} \times 4.5 \mathrm{~cm}$, and the stiffness of the fabric is tested by the SDLATLAS stiffness tester.

The fabric is measured 5 times in the warp and weft direction and the average is taken. The fabric stiffness L calculated as follows. The smaller the stiffness L value, the softer the fabric feels.

$$
L=\sqrt{\frac{\left(T^{2}+W^{2}\right)}{2}}
$$

Where, $\mathrm{L}$ - fabric stiffness $(\mathrm{cm}), \mathrm{T}$-average value in the warp direction $(\mathrm{cm}), \mathrm{W}-$ average value in the weft direction $(\mathrm{cm})$, respectively.

\section{Results and discussion}

3.1 Preparation of acrylate-terminated polysiloxane

In this experiment, a ring-opening reaction of epoxy-terminated polysiloxane and 
acrylic acid was used to synthesize acrylate-terminated polysiloxane. The reaction equation is shown in Fig 3, and the infrared spectrum of the synthesized product is shown in Fig 4.

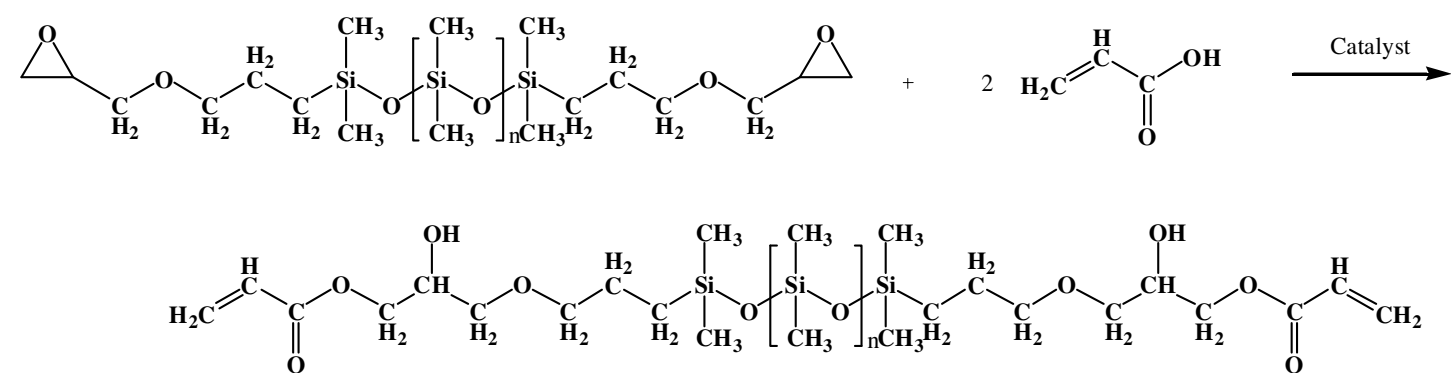

Fig. 3 ring-opening reaction equation of epoxy-terminated polysiloxane

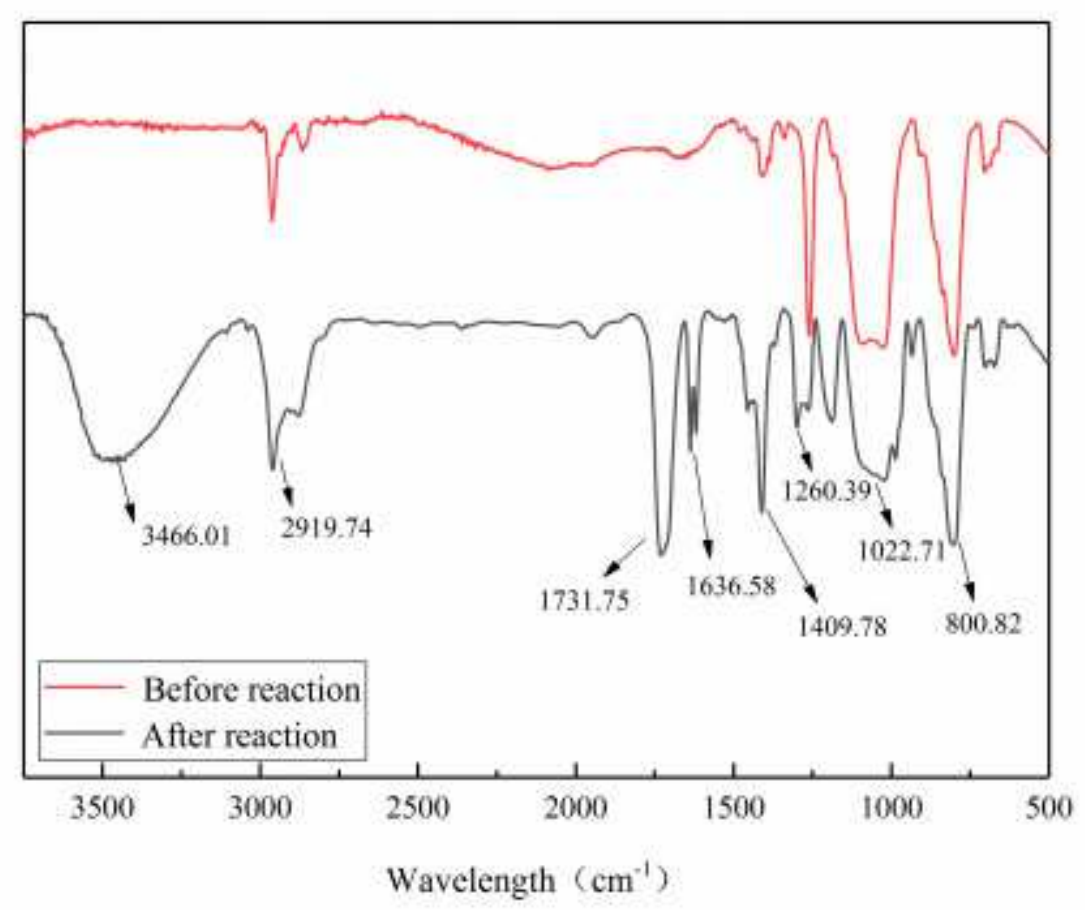

Fig. 4 FTIR spectra of epoxy-terminated polysiloxane and its ring-opening products

As shown in Fig 4, in the infrared spectrum of the epoxy-terminated polysiloxane, the stretching vibration peak of Si-O-Si is at $1022.71 \mathrm{~cm}^{-1} .1260 .39 \mathrm{~cm}^{-1}$ and 800.82 $\mathrm{cm}^{-1}$ are the stretching vibration absorption peaks of $\mathrm{Si}-\mathrm{CH}_{3}$ on the polysiloxane chain. The acrylate-terminated polysiloxane produced has a stretching vibration frequency of $-\mathrm{OH}$ at $3466.01 \mathrm{~cm}^{-1}$. The stretching vibration frequency of $\mathrm{C}=\mathrm{C}$ generally appears around $3050 \mathrm{~cm}^{-1}$, but the absorption peak is weak and is hidden by the absorption peak of the hydroxyl group, where as the stretching vibration peak at $1636.58 \mathrm{~cm}^{-1}$ indicates the existence of $\mathrm{C}=\mathrm{C}$. The absorption peak at $1731.75 \mathrm{~cm}^{-1}$ is attributed to the $\mathrm{C}=\mathrm{O}$ 
stretching vibration, which indicates the acrylate-terminated polysiloxane was synthesized.

3.2 Effect of the dosage of acrylate-terminated polysiloxane on the performance of cationic core/shell polysiloxane acrylate emulsion

This experiment uses acrylate-terminated polysiloxane ( synthesized in Section 3.1) as raw material. The $\mathrm{T}_{\mathrm{g}}$ of the mixed acrylate shell monomer is $-10^{\circ} \mathrm{C}$. The process of cationic silane-modified core/shell emulsion polymerization is described in Section 2.3. The experimental results are shown in Table1

Table 1 Effect of acrylate-terminated polysiloxane content on emulsion performance

\begin{tabular}{|c|c|c|c|c|c|}
\hline $\begin{array}{c}\text { Dosage of } \\
\text { acrylate- } \\
\text { terminated } \\
\text { polysiloxane } \\
(\%)\end{array}$ & $\begin{array}{l}\text { Emulsion } \\
\text { appearance }\end{array}$ & $\begin{array}{l}\text { Dilution } \\
\text { stability }\end{array}$ & $\begin{array}{l}\text { Particle } \\
\text { size } \\
(\mathrm{nm})\end{array}$ & $\begin{array}{c}\text { Monomer } \\
\text { conversion } \\
(\%)\end{array}$ & $\begin{array}{c}\text { Zeta } \\
\text { potential } \\
(\mathrm{mv})\end{array}$ \\
\hline 0 & milky blue & stable & 100.2 & 96.13 & 61.4 \\
\hline 5 & milky blue & stable & 98.9 & 95.61 & 62.3 \\
\hline 10 & milky blue & stable & 99.5 & 94.73 & 50.8 \\
\hline 15 & milky blue & stable & 108.7 & 93.53 & 48.9 \\
\hline 20 & milky blue & stable & 110.6 & 91.11 & 36.1 \\
\hline
\end{tabular}

It can be seen from Table 1 that with or without the added of acrylate-terminated polysiloxane, the appearance of the emulsions is all milky blue, and the emulsion can be stored for a long time without precipitation and separation. When adding acrylateterminated polysiloxane, the particle size of the emulsion increased from $\mathrm{d}=98.9 \mathrm{~nm}$ to $\mathrm{d}=110.6 \mathrm{~nm}$. The results show that the experimentally synthesized polysiloxane acrylate emulsion with different acrylate-terminated polysiloxane content has the characteristics of nano emulsion and emulsion stability.

With the increase in the dosage of acrylate-terminated polysiloxane, the 
conversion rate of the reaction monomers tends to decrease. This is because the polysiloxane chain has the characteristics of high flexibility and low surface energy. When emulsifying in water, the interfacial tension between the polysiloxane and water is relatively large, so the emulsification of the polysiloxane is relatively difficult. Under the same emulsification conditions, the stability of the emulsion system decreases as the acrylate-terminated polysiloxane content of the system increases; larger emulsified or dispersed particles of acrylate-terminated polysiloxane will appear in the emulsion. During the polymerization reaction, unstable latex particles or agglomerates are easily formed, which are filtered and removed from the emulsion after the reaction, so the monomer conversion of the emulsion system decreases. By measuring the zeta potential for the emulsion, it is found that the synthesized latex particles all have a positive potential, indicating that the synthesized latex is a cationic emulsion. The zeta potential for the synthesized latex particles gradually decreases from the increase in the addition of acrylate-terminated polysiloxane, and the electrostatic repulsion between the latex particles decreases and the latex particles are prone to collision and agglomeration under the action of mechanical shear. Thus, this is another reason for the reduction of monomer conversion.

\subsection{Morphology of latex particles}

In this work, the polymerized emulsion sample containing $15 \%$ acrylate polysiloxane was used as the material, and the internal structure of the emulsion particles was observed with a transmission electron microscope, which is shown in Fig 5. 


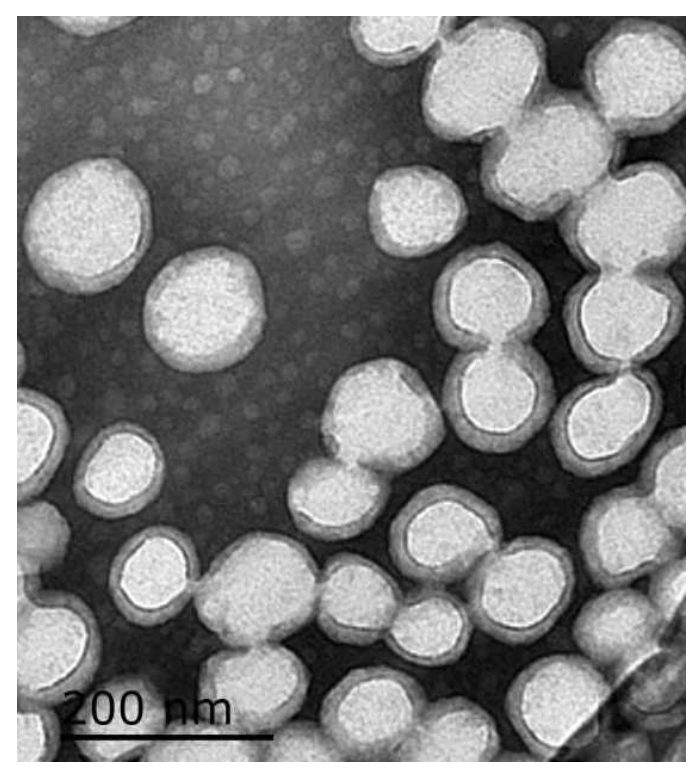

Fig.5 TEM image of core/shell cationic silicone acrylic emulsion

It can be seen from Fig 5 that the latex particles of the experimentally synthesized cationic core/shell polysiloxane acrylate emulsion has a regular spherical structure. The particle size of the latex particles is similar, and the average particle size is about $100 \mathrm{~nm}$. It shows that the nano-level core/shell polysiloxane acrylate emulsion was synthesized by this experiment.

It is evident that each latex particle of the synthesized cationic core/shell polysiloxane acrylate emulsion has two color areas, with a light-colored area at the center with a dark rim at the periphery. This is due to the difference in the composition of the elements in the latex particles, which makes the obvious gray-scale differences appear in the transmission electron microscope image. This phenomenon shows that the synthesized latex particles have an obvious core/shell structure, in which the lightcolored area in the center of the latex particle is mainly the area containing more polysiloxane segments, and the darker-colored shell area is the polyacrylate chain area. From the perspective of synthesis, although the Fig shows that there is a clear boundary of the shell and the core, there is still a covalent linkage between the core and the shell. Because the end of the polysiloxane has two reactive acrylate structures, it can be covalently bonded with the acrylate chain in the shell structure.

At the same time, the core/shell polysiloxane acrylate latex particles synthesized in this experiment have a different particle fusion phenomenon during the drying 
process than the conventional emulsion particles. The main manifestation is that when two core/shell polysiloxane acrylate latex particles get close due to dehydration and drying, the latex particles tend to merge into the core-core structure and the shell-shell structures due to the difference in elements in the core/shell structure. In different areas in Fig 5, this core-core fusion channel can be found, indicating that this fusion phenomenon of latex particles is common. It is because the core region of the latex particles is a low surface energy region with polysiloxane chains as the main component, while the shell region is a high surface energy acrylate macromolecular chain region. Due to the difference in surface energy between the core and shell, the dehydration filmforming method of the two latex particles by the way of core-core fusion and shell-shell fusion makes the membrane system in a stable state with low system energy.

3.4 Film element analysis and Hygroscopicity analysis of cationic core/shell polysiloxane acrylate emulsion

In this experiment, the film was prepared with the cationic core/shell polysiloxane acrylate emulsion synthesized in Section 3.2 as the raw material, the surface element analysis and contact angle test results of the film are shown in Table 2, and the water absorption test results of the film are shown in Fig. 6a.

Table 2 Surface contact angle and element analysis of cationic silicon modified core/shell acrylate emulsion film

\begin{tabular}{|c|c|c|c|c|c|}
\hline Dosage of & contact angle & \multicolumn{4}{|c|}{ Element content $(\%)$} \\
\hline acrylate- & $\left({ }^{\circ}\right)$ & \multirow{3}{*}{$\mathrm{C}$} & \multirow{3}{*}{$\mathrm{O}$} & \multirow{3}{*}{$\mathrm{Si}$} & \multirow[t]{3}{*}{$\mathrm{Si} / \mathrm{C}$} \\
\hline polysiloxane & & & & & \\
\hline$(\%)$ & & & & & \\
\hline 0 & 31.7 & 75.76 & 24.24 & 0 & 0 \\
\hline 5 & 39.3 & 78.73 & 18.62 & 2.65 & 0.034 \\
\hline 10 & 45.1 & 70.82 & 22.37 & 6.81 & 0.096 \\
\hline
\end{tabular}




\begin{tabular}{llllll}
\hline 15 & 64.69 & 25.9 & 9.41 & 0.15 \\
20 & 69.3 & 62.11 & 26.91 & 10.98 & 0.18 \\
\hline
\end{tabular}

It can be seen from Table 2 that the water contact angle of the comparative polyacrylate latex film is $31.7^{\circ}$, which indicates that the pure polyacrylate film has higher hydrophilicity. This is attributed to the presence of amide groups, ester groups, and water-soluble emulsifiers in the latex film. As the dosage of acrylate-terminated polysiloxane in the core/shell emulsion increases, the contact angle of the film gradually increases, indicating that the hydrophobicity of the film increases. When the addition amount of acrylate-terminated polysiloxane is $20 \%$, the water contacts angle of the film reaches the maximum $69.3^{\circ}$.

This is because in latex the two latex particles first appear to be the core-core and shell-shell fusion phenomena during the drying process, and the core-core fusion area and the shell-shell fusion area gradually increase; in order to obtain the stability of the film structure, the difference in surface energy between the core/ shell causes the corecore fusion area to be inverted to the surface of the film under certain conditions, That means part of polysiloxane chains migrate and gather on the surface of the film. Therefore, the hydrophobicity of the membrane surface increases, and its water contacts angle increases. This phenomenon is also confirmed by the surface atomic energy spectrum data in Table 2. As the dosage of acrylate-terminated polysiloxane in the core/shell emulsion increases, the $\mathrm{Si} / \mathrm{C}$ value on the surface of the film also increases (from 0.034 to 0.18 ).
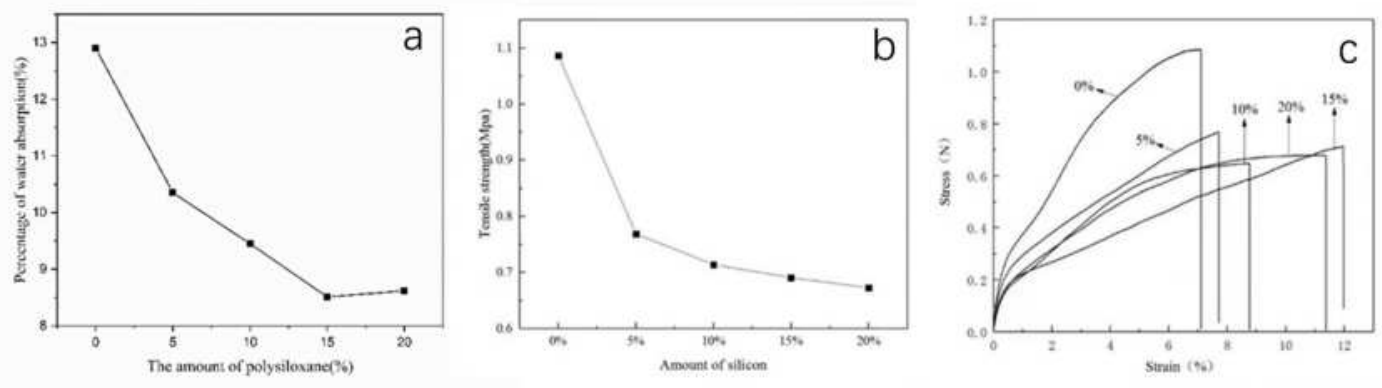
Fig. 6. a) Hygroscopicity of cationic silicon modified acrylate emulsion film; b) Effect of acrylate-terminated polysiloxane dosage on the fracture strength of emulsion films; c) Effect of acrylate-terminated polysiloxane dosage on the stress-strain curve relationship of emulsified films

It can be seen from Fig $6 a$ that the moisture absorption rate of the comparative cationic acrylate emulsion film is $12.89 \%$, and the moisture rate of the cationic core/shell polysiloxane acrylate latex film gradually decreases with the dosage of terminal acrylate silane increased. When the terminal acrylate silane dosage is $15 \%$, the moisture absorption rate reaches the lowest value.

The analysis shows that there are two kinds of performance macromolecular segments in the polymer film. Among them, the macromolecular segment of polyacrylate contains a large number of ester groups and amide groups. These groups have high polarity and can form hydrogen bonds with water molecules, so that the polyacrylate chain has a certain hygroscopicity. On the contrary, each silicon atom in the polysiloxane chain has two weakly polar methyl substituents. The oxygen atom between the two silicon atoms in the main chain is sterically hindered, making it difficult to interact with water molecules by hydrogen bonds, so the polysiloxane chain does not have hygroscopic properties. When the content of polysiloxane chains in the polymer film increases, the hygroscopicity of the entire film will decrease.

At the same time, it can be seen from Table 2 that when the dosage of acrylateterminated silane increases, the silicon content on the surface of the film increases, the contact angle of water increases, and the hydrophobicity of the polymer film surface is enhanced. Therefore, the presence of polysiloxane chains on the surface of the film plays a role in hindering the absorption and diffusion of water molecules. Hence, increasing the dosage of acrylate-terminated silane in the cationic core/shell polysiloxane acrylate emulsion can effectively reduce the hygroscopicity of the film. 
3.5 Film mechanical properties of cationic core/shell polysiloxane acrylate emulsions

This experiment uses the cationic core/shell polysiloxane acrylate emulsions synthetized in Section 3.2 as the raw material to study the effect of the dosage of acrylate-terminated siloxane on the mechanical properties of the polymer emulsion film. The results are shown in Fig 6b-c. Increasing the dosage of acrylate-terminated polysiloxane in the polymerization emulsion, the breaking strength of the film shows a decreasing trend (Fig 6b), while the breaking elongation of the film shows an increasing trend (except for $20 \%$ samples) (Fig 6c).

In the comparison sample $(0 \%)$, the polymer film is composed of polyacrylate macromolecular chains, and the ester groups and amide groups on the macromolecular chains can form hydrogen bonds, dipole forces, and other intermolecular interaction forces. At the same time, a certain crystalline area is formed in film (Fig 7). All these factors can enhance the interaction between macromolecular chains and improve the ability of the membrane to resist external forces to produce deformation.

When the polysiloxane chain is introduced into the polymer film, since there is no polar group on the polysiloxane chain and its specific surface energy is low, nano aggregate structures of the polysiloxane chain are formed and dispersed in the film structure, and part of the polysiloxane chains reversely migrate to the surface of the film. Since the interaction force between the polysiloxane chains and the polyacrylate chain is weak, the polysiloxane chain nano aggregates in the film structure become the weak point of the mechanical behavior of the film structure, it will become a stress concentration points under the external stress so the breaking strength of the film decreases. When the quantity of acrylate-terminated polysiloxane in the film increases, the weaker points of stress concentration increase, and the breaking strength of the film will be reduced further.

Since the acrylate-terminated polysiloxane chain and the acrylate macromolecular main chain are not simply physically mixed, they are bonded together by covalent bonds. The introduction of acrylate-terminated polysiloxane chain can increase the molecular weight and network structure of the polymer, so when the polymer film is 
stretched and deformed by an external force, the polysiloxane chains can slip along with the acrylate macromolecular chains, the film's broken elongation increases, the tensile modulus of the film decreases, and the polymer film becomes soft in handle.

3.6 The influence of cationic core/shell polysiloxane acrylate emulsion on the pigment pad dyeing of cotton fabric

The cationic core/shell polysiloxane acrylate emulsion in Section 3.2 was used as a binder for the pigment dyeing of cotton fabrics to study the influence of the binder on the pigment dyeing performance. The pigment dyeing process is as described in Section 2.4 with the binder dosage is $25 \mathrm{~g} / \mathrm{L}$, and the experimental results are shown in Table 3 , schematic image of pigment dyeing is shown in Fig. 7

Table 3 Effect of the dosage of acrylate-terminated polysiloxane on the color fastness of pigment(B31) dyed fabric

\begin{tabular}{|c|c|c|c|c|c|c|}
\hline \multirow{2}{*}{$\begin{array}{c}\text { Dosage of } \\
\text { acrylate- } \\
\text { terminated } \\
\text { polysiloxane } \\
\text { (\%) }\end{array}$} & \multicolumn{2}{|c|}{ Rubbing fastness (grade) } & \multicolumn{2}{|c|}{$\begin{array}{c}\text { Soaping } \\
\text { fastness (grade) }\end{array}$} & \multirow{2}{*}{$\mathrm{K} / \mathrm{S}$} & \multirow{2}{*}{$\begin{array}{c}\text { Hardness } \\
(\mathrm{cm})\end{array}$} \\
\hline & Dry & Wet & $\begin{array}{l}\text { Color } \\
\text { fading }\end{array}$ & $\begin{array}{l}\text { Color } \\
\text { staining }\end{array}$ & & \\
\hline 0 & $3 \sim 4$ & 3 & 4 & $4 \sim 5$ & 1.6 & 3.5 \\
\hline 5 & 4 & 3 & $4 \sim 5$ & $4 \sim 5$ & 1.8 & 3.4 \\
\hline 10 & 4 & $3 \sim 4$ & $4 \sim 5$ & $4 \sim 5$ & 1.6 & 3.3 \\
\hline 15 & $4 \sim 5$ & 4 & $4 \sim 5$ & $4 \sim 5$ & 1.8 & 3.3 \\
\hline 20 & $4 \sim 5$ & 4 & 4 & $4 \sim 5$ & 1.8 & 3.2 \\
\hline
\end{tabular}




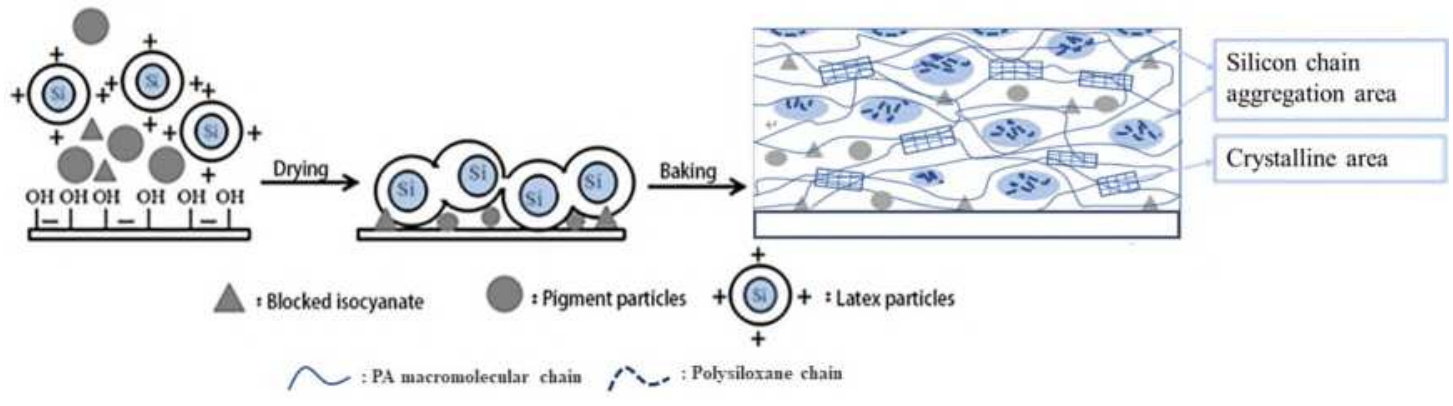

Fig. 7 Schematic image of pigment dyeing

As shown in Table 3, compared with polyacrylate emulsion, the soaping fastness of cotton fabrics pigment dyed with cationic core/shell polysiloxane acrylate emulsions as a binder basically remains unchanged at grade 4 , and the rubbing fastness (wet/dry) of pigment-dyed cotton fabric are between grade 3 and grade 4 5. It shows that using the core/shell polysiloxane acrylate emulsion synthesized in this experiment as a pigment dyeing binders can obtain dyed fabrics that meet the fastness requirements of the national standard. This result indicates that the experimentally synthesized polymer emulsion can form a polymer film with good bonding fastness on the fiber surface. As the baking process, the blocked isocyanate is unblocked under high temperature conditions, a three-dimensional network film formed by the hydroxyl group in the binder and the hydroxyl group on the cotton fiber with covalent cross-linking reaction make the pigment particles adhered to the fiber surface firmly. Therefore, both soaping fastness and dry/wet rubbing fastness are all improved.

There is no obvious change in the K/S value data in Table 3, which indicates that after the core/shell silicone acrylic emulsion is dried into a film, the polysiloxane chains cannot produce a continuous and low refractive index polysiloxane film on the film surface (Chengbing et al. 2019), so the deepening effect on pigment dyed cotton fabric is not obtained.

It also can be seen from Table 3 that the softness of pigment-dyed fabrics will increase with the increase of the quantity of acrylate-terminated polysiloxane in the emulsion. This is because after the core/shell polysiloxane acrylate emulsion forms a film on the surface of the cotton fiber, the surface of the film gathers a large number of polysiloxane chains (Fig 7), which reduces the frictional resistance between the fiber 
and the fabric, so the fabric is easy to deform under the action of external force, and has a softer feel.

3.7 Effect of the dosage of cationic core/shell polysiloxane acrylate emulsion on the fastness of dyed fabrics

The pigment dyeing process is as described in Section 2.4. Select the cationic core/shell polysiloxane acrylate emulsion with $15 \%$ acrylate-terminated polysiloxane content as a binder to explore the effect of the dosage of binders in the pigment dye solution on the dyeing performance of dyed fabrics. The results are shown in Table 4:

Table4 Effect of binder dosage on the properties of the dyed fabric

\begin{tabular}{ccccccc}
\hline \multirow{2}{*}{$\begin{array}{c}\text { Binder } \\
\text { agent } \\
\text { content } \\
\text { (g/L) }\end{array}$} & Rubbing fastness (grade) & \multicolumn{2}{c}{$\begin{array}{c}\text { Soaping fastness } \\
\text { (grade) }\end{array}$} & K/S & $\begin{array}{c}\text { Hardness } \\
\text { (cm) }\end{array}$ \\
\cline { 2 - 5 } 0 & 0 & Wet & $\begin{array}{c}\text { Color } \\
\text { fading }\end{array}$ & $\begin{array}{c}\text { Color } \\
\text { staining }\end{array}$ & & \\
\hline 10 & 3 & 3 & 0 & 0 & 0 & 2.5 \\
15 & 4 & 3 & $4 \sim 5$ & $4 \sim 5$ & 2.1 & 2.9 \\
20 & 4 & $3 \sim 4$ & $4 \sim 5$ & $4 \sim 5$ & 1.8 & 3.1 \\
25 & $4 \sim 5$ & 4 & $4 \sim 5$ & $4 \sim 5$ & 1.4 & 3.2 \\
30 & $4 \sim 5$ & 4 & $4 \sim 5$ & $4 \sim 5$ & 1.2 & 3.5 \\
\hline
\end{tabular}

As shown in Table 4, the dry/wet rubbing fastness and soaping fastness of the dyed fabric increase first and then stabilize with the increase of the dosage of binders in the dye solution. When the dosage of binder is $25 \mathrm{~g} / \mathrm{L}$ and $30 \mathrm{~g} / \mathrm{L}$, the dry/wet rubbing fastness basically unchanged. When the dosage of binder is greater than $15 \mathrm{~g} / \mathrm{L}$, the soaping fastness remains unchanged.

Increasing the dosage of the binder can increase the thickness of emulsion film on the pigment particles, thereby improving the fastness of the dyed fabric. When the dosage of the binder is $25 \mathrm{~g} / \mathrm{L}$, the dyed fabric has good dry/wet rubbing fastness, of 
which dry rubbing fastness reaches grade $4 \sim 5$, wet rubbing fastness reaches grade 4 , soaping fastness in fade and stain fastness reach grade 4 5, and the fabric feels soft. Continue to increase the dosage of binder, the film thickness of the fabric surface will increase. At the same time, it makes stiffness of the fabric increases and the hand feels becomes hard. In addition, the increase in the thickness of the film leads to an increase in the reflection of visible light by the binder film, which makes the K/S value reduced.

\subsection{Effect of baking temperature on dyeing performance}

The pigment dyeing process is as described in section 2.4. The silicone-acrylic emulsion binder with a silicon content of $15 \%$ was selected as the binder, and the dosage of the binder in the pigment dyeing solution was $25 \mathrm{~g} / \mathrm{L}$. The effect of baking temperature on dyeing performance was explored. The results are shown in Table 5:

Table 5 Effect of baking temperature on the properties of dyed fabrics

\begin{tabular}{|c|c|c|c|c|c|c|}
\hline \multirow{2}{*}{$\begin{array}{c}\text { Baking } \\
\text { temperature } \\
\left({ }^{\circ} \mathrm{C}\right)\end{array}$} & \multicolumn{2}{|c|}{ Rubbing fastness (grade) } & \multicolumn{2}{|c|}{$\begin{array}{l}\text { Soaping fastness } \\
\text { (grade) }\end{array}$} & \multirow{2}{*}{$\mathrm{K} / \mathrm{S}$} & \multirow{2}{*}{$\begin{array}{l}\text { Hardness } \\
(\mathrm{cm})\end{array}$} \\
\hline & Dry & Wet & $\begin{array}{l}\text { Color } \\
\text { fading }\end{array}$ & $\begin{array}{l}\text { Color } \\
\text { staining }\end{array}$ & & \\
\hline 100 & 3 & 3 & $3 \sim 4$ & $3 \sim 4$ & 1.5 & 2.9 \\
\hline 110 & 3 & 3 & 4 & 4 & 1.4 & 2.9 \\
\hline 120 & $3 \sim 4$ & 3 & 4 & 4 & 1.3 & 3.1 \\
\hline 130 & 4 & $3 \sim 4$ & $4 \sim 5$ & $4 \sim 5$ & 1.5 & 3.1 \\
\hline 140 & $4 \sim 5$ & 4 & $4 \sim 5$ & $4 \sim 5$ & 1.4 & 3.2 \\
\hline 150 & $4 \sim 5$ & 4 & $4 \sim 5$ & $4 \sim 5$ & 1.4 & 3.4 \\
\hline
\end{tabular}

As shown in Table 5, when the baking temperature increases, the K/S value is basically unchanged, and the dry/wet rubbing fastness and soaping fastness of the dyed fabric is improved, but the softness of the fabric is decreased.

In the baking process, the crosslink reaction of the blocked isocyanate, binders and the cotton fabric are insufficient for the lower baking temperature conditions, so 
the color fastness of the fabric is poor. As the baking temperature rises, the crosslink of the blocked isocyanate is fully carried out, and the pigment particles are firmly adhered to the surface of the fabric, so the soaping fastness and the dry/wet rubbing fastness are improved. But when the baking temperature exceeds $140^{\circ} \mathrm{C}$, the full crosslinking between the binder and the cotton fiber causes the fabric to feel harder.

\subsection{Analysis of fabric appearance before and after pigment dyeing}

The SEM images of the fabric before and after pigment dyeing were compared (the dosage of binder was $25 \mathrm{~g} / \mathrm{L}$, the baking temperature was $140{ }^{\circ} \mathrm{C}$ ) as shown in Fig. 8.
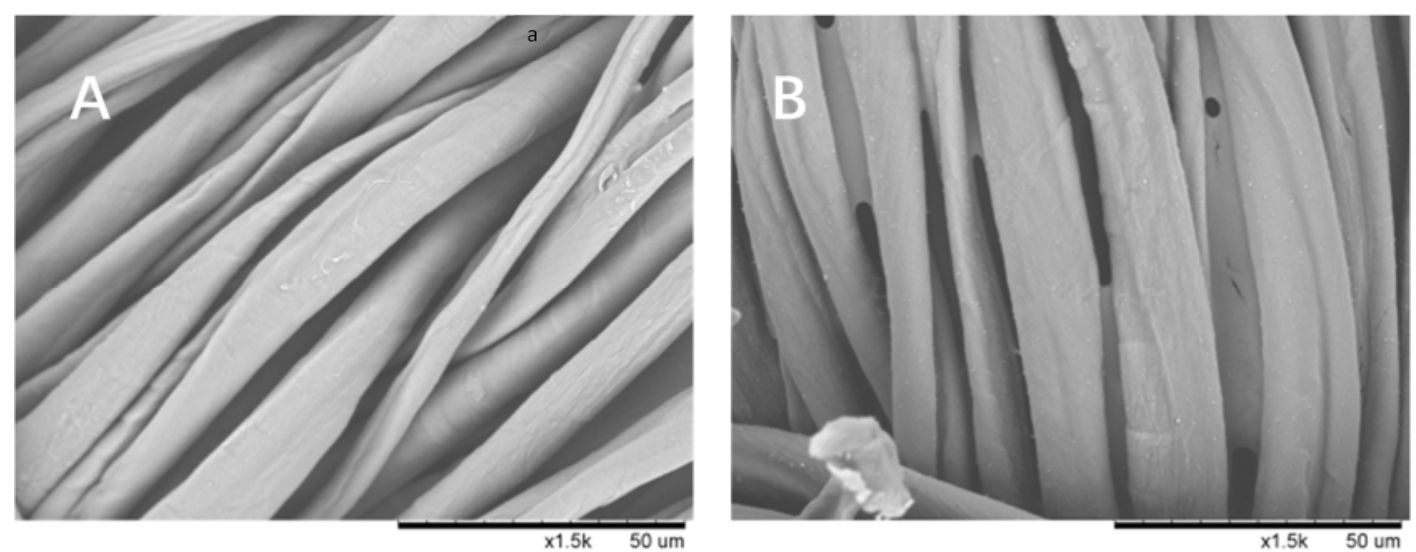

Fig.8 SEM image of pigment dyed fabric

A: undyed cotton fabric, B: cotton fabric after pigment dyeing

As shown in Fig 8, the appearance of cotton fiber did not change significantly before and after dyeing. There is a layer of smooth film structure on the surface of cotton fiber dyed with pigment. There are also membrane adhesion structures between fiber. No aggregation of pigment particles or uneven accumulation of binders is observed on the surface of the fiber, which indicates that the cationic polymer emulsions can form a uniform membrane structure on the surface of the cotton fiber through the adsorption of the electrostatic charge between the fiber and the latex particles. 


\section{Conclusion}

(1) In this article, acrylate-terminated polysiloxane was synthesized and successfully used to prepare a nano-scale cationic core/shell polysiloxane acrylate emulsion. The average size of the latex particles is about $100 \mathrm{~nm}$ and the emulsion has a good stability. (2) During the film-forming process of the cationic core/shell polysiloxane acrylate emulsion, there is a core-core and shell-shell fusion phenomenon between the latex particles, and polysiloxane chains accumulate on the surface of the film and the remaining polysiloxane chains are dispersed in the membrane structure in the form of nano aggregates.

(3) Cationic core/shell polysiloxane acrylate emulsion can be used as a pigment dyeing binder for cotton fabric, which will allow dyed fabrics with good soft hand feeling, dry/wet rubbing fastness of grade $4 \sim 5$, and soaping fastness of grade 4 , which meet national standards.

Conflict of Interest: The authors declare that they have no conflict of interest.

This article does not contain any studies with human participants or animals performed by any of the authors. Informed consent was obtained from all individual participants included in the study.

Acknowledgment: No funding was received.

\section{References}

C X Wang HQW, etal (2009) Dyeing of Superfine Coating Tube Yarn Dyeing\&Finishing 35:23-25

Chengbing Y, Yilin L, Yixun C, Dan Y (2019) Poly(methyl methacrylate)/polysiloxane core-shell latex as a deepening agent for dyed polyester fabric Coloration Technology 135:46-53

F Y Shen KJF (2009) Application of Cationic Emulsion Adhesive in Coating Rolling and Dyeing Dyeing\&Finishing 8:6-9

Fang K ZL, Xu Y, et al (2010) Pigment Dyeing of Polyamide-Epichlorohydrin 
Cationized Cotton Fabrics Journal of Applied Polymer Science 118:2736-2742

Fu S, Luo X, Yao D, Tian A, Wang C (2013) Effect of pigment particle character on dyeing performance of cotton fabrics Fibers and Polymers 14:1019-1023

H Li ZhZ (2013) Review of core-shell acrylate pigment printing adhesive Textile Dyeing and Finishing Journa 10:4-9

Ishwarlal RA (2000) Method of dyeing anionic materials with pigment colors having a net cationic charge using a padding process. US Patent US6080687 A,

J L Ji FJ, C Zhang (2009) Preparation of cationic binder for pigment dyeing Dyeing\&Finishing 35:9-12+20

J M Xia ZJZ, etal (1996) Development and application of cationic modifier ZF Journal of printing and dyeing auxiliaries 7:24-29

Jie Z (2005) Problems in Coating Printing Technology Textile Information Weekly 6:14-14

Lewis DM, Mcllroy KA (2010) The chemical modification of cellulosic fibres to enhance dyeability Coloration Technology 27:5-17

Martini T, Keil KH, Karsunky U, Sternberger K (1991) Dyeing textile material with pigment dyes: pre-treatment with quaternary ally ammonium salt polymer. US,

Martini T, Keil KH, Karsunky U, Sternberger K (1993) Process for dyeing textile materials with pigment dyes. EP,

Song XY (2013a) Dyeing of Coatings and Energy Saving and Emission Reduction ( I ) Dyeing\&Finishing 039:44-47 doi:CNKI:SUN:YIRA.0.2013-12-013

Song XY (2013b) Dyeing of Coatings and Energy Saving and Emission Reduction ( II ) Dyeing\&Finishing 39:42-44 doi:10.3969/j.issn.1000-4017.2013.14.015

Song XY (2013c) Dyeing of Coatings and Energy Saving and Emission Reduction ( III ) Dyeing\&Finishing 39:43-47 doi:CNKI:SUN:YIRA.0.2013-14-015

Song ZSJ (2015) Preparation of core-shell organosilicon-acrylate emulsion as pigment printing adhesive Shanghai Textile Science \& Technology 43:74-78

Sui Z, Pang W, Song J, Cao X (2013) Synthesis and Properties of Pigment Printing Adhesive with Core-Shell Structure for Textiles Asian Journal of Chemistry 25:10515-10518

Sun G, Li. D (2007) Dyeing textiles using nanoparticles. US Patent US7048771 B2,

Tong Z (2012) Discussion on determination method of epoxy value of epoxy terminated silicone oil Silicone Materials 26:258-261

Womble GF (1952) Dyeing with pigment coloring matter. US,

Wu X (2016) Preparation by Emulsion Polymerization and Application in Pigment Dyeing of Polyacrylate Adhesive Journal of Zhongyuan University of Technology 27:42-46

Wu Y, Zhang ZQ (2013) Application of Cationic Modification Agent to Pigment Dyeing Advanced Materials Research 627:369-373

Xu HZM (2009) Application performances of ultra-fine cationic pigment on cotton dip dyeing Dyeing\&Finishing 35:1-4

Yaman N OE, Seventekin N (2012) Improvement Fastnesses and Color Strength Of Pigment Printed Textile Fabric Journal of Engineered Fabrics \& Fibers 7:40-47 
